\title{
PAPER \\ Support by Warning or by Action: Which is Appropriate under Mismatches between Driver Intent and Traffic Conditions?
}

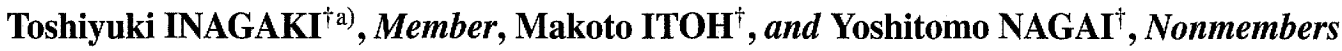

SUMMARY This paper tries to answer the following question: What type of support should be given to an automobile driver when it is determined, via some method to monitor the driver's behavior and the traffic environment, that the driver's intent may not be appropriate to a traffic condition? With a medium fidelity, moving-base driving simulator, three conditions were compared: (a) Warning type support in which an auditory warning is given to the driver to enhance his/her situation recognition, (b) action type support in which an autonomous safety control action is executed to avoid an accident, and (c) the baseline condition in which no driver support is given. Results were as follows: (1) Either type of driver support was effective in accident prevention. (2) Acceptance of driver support functions varied context dependently. (3) Participants accepted a systeminitiated automation invocation as long as no automation surprises were possible to occur.

key words: proactive safety, intelligent driver support, adaptive automation, levels of automation

\section{Introduction}

In the classic study of the causes of traffic accidents, Treat et al. [1] ascribe $92.6 \%$ of car accidents to human error, such as, improper lookout, inattention, and internal or external distraction. The situation is still basically the same [2]. Driving a car requires a continuous process of perception, decision, and action. Understanding of the current situation determines what action needs to be done [3]. In reality, however, a driver's situation understanding may not always be perfect. Decisions and actions following poor or imperfect situation understanding can never match a given traffic condition.

The driver's situation recognition is not directly observable. However, monitoring the driver's behavior and traffic condition may make it possible to detect mismatches between his/her intent and the traffic condition. Various efforts have thus been made to develop driver behavior monitoring methods [4]-[12].

Now a research question arises. Suppose that some monitoring method has determined that a driver may not be aware of a threat. What type of support should be given to the driver? Setting off a warning to the driver? Or, letting the support system execute an autonomous action for avoiding the threat? Let us call the former $a$ warning type support, and call the latter an action type support.

Manuscript received January 9, 2007.

Manuscript revised June 8, 2007.

Final manuscript received August 8, 2007.

${ }^{\dagger}$ The authors are with the Department of Risk Engineering, University of Tsukuba, Tsukuba-shi, 305-8573 Japan.

a) E-mail: inagaki@ risk.tsukuba.ac.jp

DOI: $10.1093 /$ ietfec/e90-a.11.2540
The warning type support adopts an approach to enhance the driver's situation awareness, expecting that, once the driver succeeds in understanding the ongoing situation, he/she will be able to determine what action needs to be done and implement it appropriately. The warning type support is compatible with the human-centered automation concept in which it is assumed that the human is maintained as the final authority [13]-[15]. However, an accident may occur if the driver (the final authority) failed or was unable to respond to the warning at the right time.

The action type support applies a system-initiated automation invocation (or, shifting of authority from the driver to automation) to prevent an accident from occurring. The system-initiated automation invocation is a basic function of adaptive automation [16], [17] that trades authority between humans and machines dynamically in time depending on the situation. Such a system-initiated trading of authority may sometimes bring negative consequences, such as automation surprises, over-trust in automation, distrust of automation (see, e.g., [18]-[21]).

Asking which type of support is better between the above two is basically the same as asking whether the human must be maintained as the final authority at all times and on every occasions (as is claimed in the human centeredautomation). An important aspect that has been sometimes overlooked is domain-dependence of human-centered automation [22]. In other words, although aviation tries to stick to the human-centered automation rather strictly [14], [23], automobile may not be a domain to which the same principle as aviation may be applicable, because quality of human operators and time-criticality are quite different between the two domains [22].

This paper reports results of an experiment with a medium fidelity, moving-base driving simulator, for investigating efficacy and driver acceptance of the warning type support and the action type support. It is argued that driver support needs to be situation-adaptive in which its level of automation is adjustable in a context-dependent manner.

\section{Method}

\subsection{Apparatus}

The experiment was conducted with a Honda research-anddevelopment-type driving simulator with six-axis motion functionalities (Fig. 1 left). A $6 \mathrm{~km}$-long beltway course on an expressway was used in the experiment. On the center of 

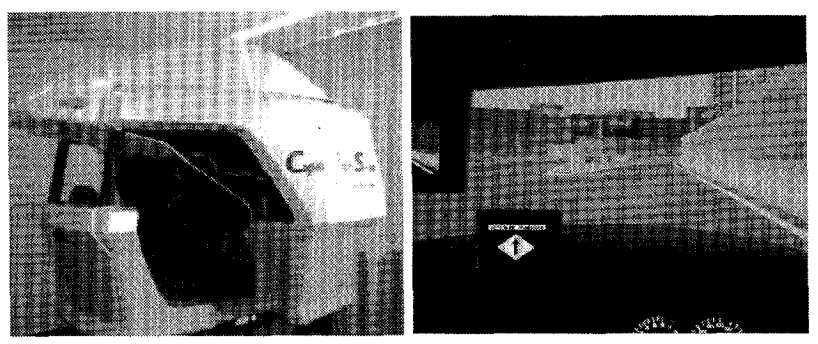

Fig. 1 Research vehicle.

the dashboard, a 7-inch display was installed to give messages and road indicators to the driver (Fig. 1 right).

\subsection{Participants and Driving Tasks}

Ten university students ( 8 males and 2 females between the ages of 21-24) driving daily participated in the experiment.

The participants were instructed to drive safely in the cruising lane at around $80 \mathrm{~km} / \mathrm{h}$ (ca. $50 \mathrm{mph}$ ) on a 2-lane expressway. The "Lane Closed" indicator appeared occasionally (see Fig. 1). The participants were allowed to enter the passing lane when the indicator was for the cruising lane. However, they were instructed to come back to the cruising lane when it became available again. When the indicator was for the passing lane, the participants had to watch for cars in the passing lane, because they might cut in to the cruising lane.

As the secondary task, every participant was requested to perform mental computations consecutively in time while driving. More concretely, a question (e.g., "Three minus five equals what?") was given every three seconds. The secondary tasks were introduced to simulate situations in which drivers may have to perform non-driving cognitive tasks that might degrade the driver's ability to understand traffic conditions, such as approaches of curves, behaviors of other vehicles. No auditory cue (such as engine sound) was given to the participants regarding other vehicles, which means that participants can not sense acoustically an approach of a faster vehicle from behind in the passing lane. They had to allocate their visual attentions intentionally to grasp positions and behaviors of other vehicles.

\subsection{Independent Variable}

Level of automation (LOA) was the within subject variable. Table 1 gives scales of LOAs, in which an LOA is added between levels 6 and 7 in the original list by Sheridan [24]. The added level, called 6.5, was first introduced in [25] to avoid automation surprises that may be induced by an automatic safety control in emergency.

Two types of support conditions as well as the no-aid baseline condition were tested.

(a) Warning type support condition, in which the system sets off an auditory proximity warning when it detects that the host vehicle is coming close to some other vehicle. The LOA of the warning type support is set at 4 .
Table 1 Scales of levels of automation [24], [25]

1. The computer offers no assistance; human must do it all.

2. The computer offers a complete set of action alternatives, and

3. narrows the selection down to a few, or

4. suggests one, and

5. executes that suggestion if the human approves, or

6. allows the human a restricted time to veto before automatic execution,

6.5 executes automatically after telling the human what it is going to do,

7. executes automatically, then necessarily informs humans, or

8. informs him after execution only if he asks, or

9. informs him after execution if it, the computer, decides to.

10. The computer decides everything and acts autonomously, ignoring the human

(b) Action type support condition, in which the system executes an appropriate safety control action automatically when it detects a hazardous event. Two kinds of safety control actions were possible in the experiment: (b-1) The system prohibits the driver from steering to change the lane when it determines that the lane change may cause a collision into a vehicle coming from behind in the passing lane, and (b-2) the system applies an automatic panic brake when it detects a rapid deceleration of the lead vehicle in the cruising lane. The LOA of such an action type support is set at 7.

(c) Baseline condition, in which no support function is available. The LOA is set at 1 in this case.

\subsection{Dependent Variables}

The following data were collected during the experiment: steering wheel angle, pressure of gas and brake pedals, operations of the turning signal, distances to other surrounding vehicles, time elapsed before the driver applied the brake $\mathrm{him} / \mathrm{herself}$, collisions into some other vehicles, velocity and position of each vehicle in the environment, subjective ratings of efficacy of the support system in accident prevention and acceptance of the support system.

\subsection{Procedure}

After receiving instructions on driving tasks, participants were given practice drives so that they could acquire familiarity with the simulator and its operation, driving environment (viz., the $6 \mathrm{~km}$-long beltway course used in the experiment), driving tasks, "Lane Closed" indicators, and support functions (viz., the warning type support and the action type). The practice drive was followed by the experimental drive in which dependent variables were measured under scenarios 1 (in which "Lane Closed" indicator is given for the cruising lane) and 2 (in which "Lane Closed" indicator is for the passing lane). 


\section{Results}

\subsection{Results under Scenario 1}

Scenario 1. The "Lane Closed" indicator appears four times for the cruising lane. Two of those four occasions are peaceful, in which there is no possibility for the host vehicle to come close to any other vehicles in the passing lane even when the host vehicle makes a lane change. In the other two occasions, however, a faster vehicle (let us call it Vehicle F) approaches from behind in the passing lane. Vehicle $F$ is in the blind spot of the host vehicle, and may not be seen easily unless the host vehicle's driver searches for such a possible threat with care. If the driver steers the wheel carelessly, the host vehicle may come too close to Vehicle F or may actually collide with it. A most sensible strategy for the host vehicle's driver in this case may be to slow down slightly, let Vehicle $\mathrm{F}$ pass the host vehicle, and then initiate steering for making a lane change.

Suppose that the driver of the host vehicle initiates to steer, without noticing the approach of a faster vehicle $\mathrm{F}$ from behind in the passing lane.

The warning type support system sets off an auditory proximity warning when it detects that the host vehicle may come close to Vehicle F.

The action type support system, on the other hand, prohibits and cancels out the steering input by the host vehicle's driver when it determines that his/her steering might cause a collision with Vehicle F. Such a system-initiated trading of authority from the driver to automation is an example of envelope protection [14] for automobile.

Under Scenario 1, each of the 10 participants firstly experienced the baseline condition in which no driver support functions were available. Five participants then made a drive under the warning type support condition, followed by a drive under the action type support condition. For the other five participants, the order of experiencing the two driver support conditions was reversed.

Results. (a) Either type of support system was effective in reducing the number of collisions with Vehicle F. Among 20 possibly hazardous occasions (viz., two occasions for each of 10 participants), 13 collisions occurred under the no-aid baseline condition, one collision under the warning type support condition, and three collisions under the action type support condition; see Table 2. The McNemar tests with STATISTICA showed highly significant differences in the number of collisions between the baseline condition and the warning type support condition $(Z=3.18, p=0.002)$, as well as between the baseline condition and the action type support condition $(Z=2.85, p=0.004)$. No significant difference was found between the warning type and the action type support conditions.

(b) Participants gave their subjective ratings on a 10point scale regarding the perceived efficacy of the support system in accident avoidance ( 1 = not effective at all, 10 = completely effective); see Fig. 2 . A Wilcoxon's signed-
Table 2 Number of collisions with Vehicle F under Scenario 1.

\begin{tabular}{cccc}
\hline & $\begin{array}{c}\text { baseline } \\
\text { (no-aid) }\end{array}$ & $\begin{array}{c}\text { warning } \\
\text { type }\end{array}$ & $\begin{array}{c}\text { action } \\
\text { type }\end{array}$ \\
\hline Collision & 13 & 1 & 3 \\
\hline No Collision & 7 & 19 & 17 \\
\hline
\end{tabular}

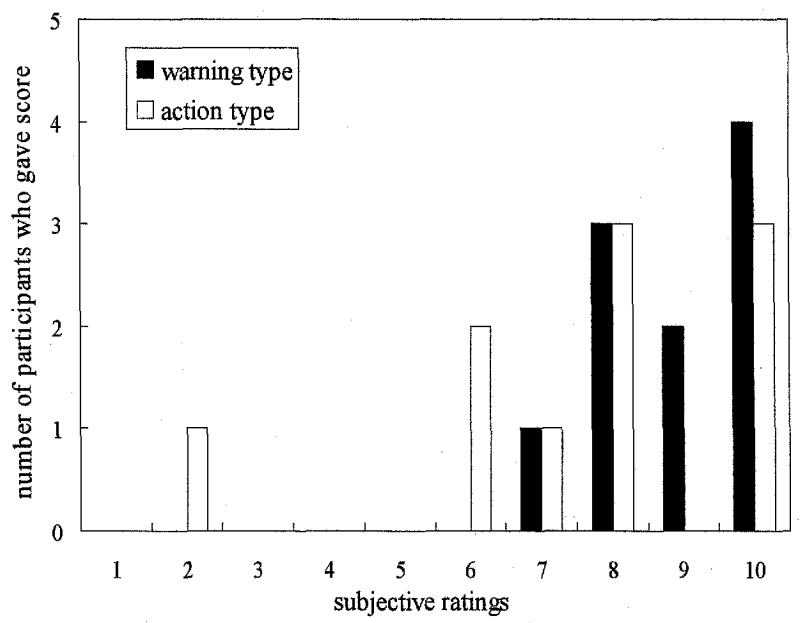

Fig. 2 Subjective ratings on perceived efficacy of the support system under Scenario 1.

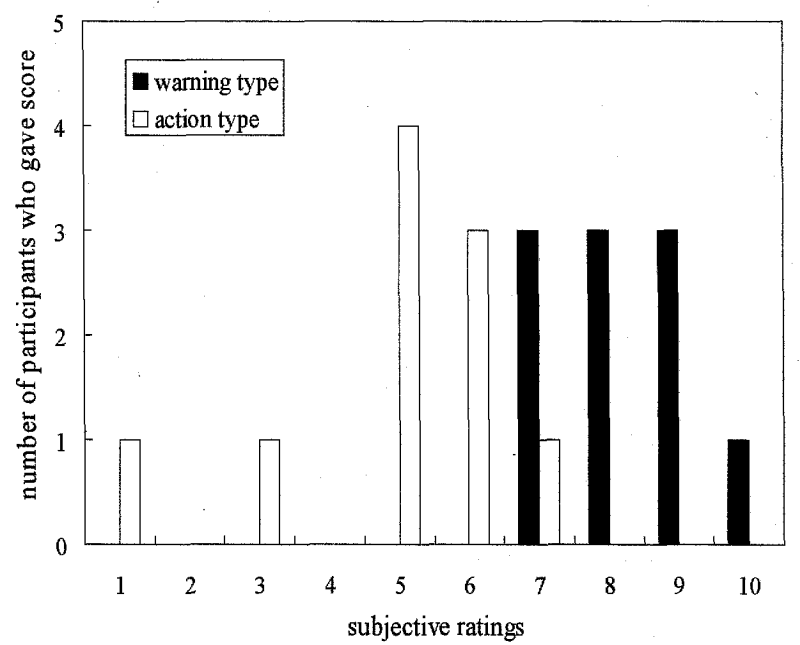

Fig.3 Subjective ratings on acceptance of the support system under Scenario 1.

rank test with STATISTICA shows no significant difference between the warning type and the action type support conditions.

(c) Participants were also requested to state their system acceptance on a 10-point scale ( 1 = not acceptable at all, $10=$ completely acceptable); see Fig. 3 . A Wilcoxon's signed-rank test shows that participants accept the warning type more than the action type $(Z=2.37, \mathrm{p}=0.018)$.

\subsection{Results under Scenario 2}

Scenario 2. For each participant, the "Lane Closed" indicator appears four times for the passing lane. In two of the four occasions, a faster vehicle (Vehicle F) in the passing 
Table 3 Number of collisions with Vehicle F under Scenario 2.

\begin{tabular}{cccc}
\hline & $\begin{array}{c}\text { baseline } \\
\text { (no-aid) }\end{array}$ & $\begin{array}{c}\text { warning } \\
\text { type }\end{array}$ & $\begin{array}{c}\text { action } \\
\text { type }\end{array}$ \\
\hline Collision & 7 & 2 & 0 \\
\hline No Collision & 13 & 18 & 20 \\
\hline
\end{tabular}

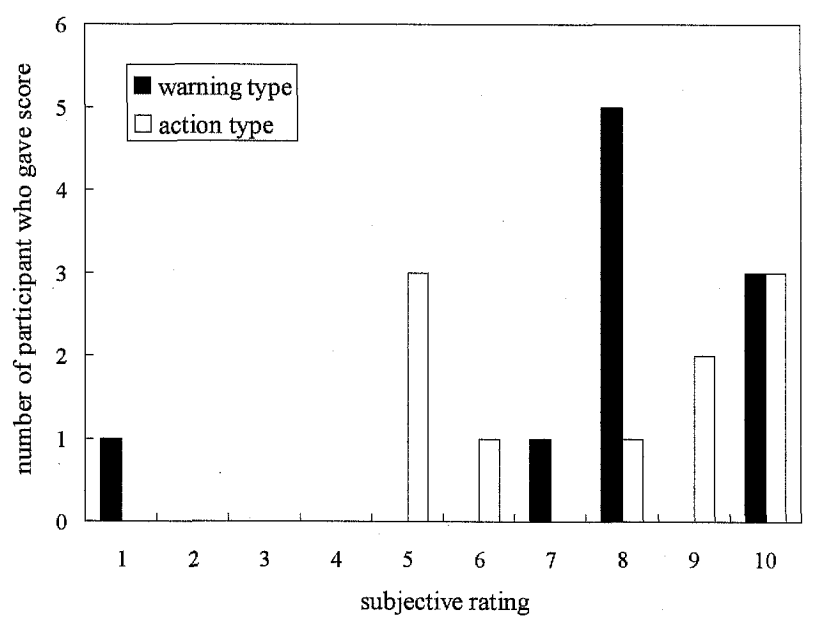

Fig. 4 Subjective ratings on perceived efficacy of the support system under Scenario 2.

lane makes a lane change in response to the indicator and cuts in between the host vehicle and its lead vehicle (Vehicle $\mathrm{L}$ ) in the cruising lane. Vehicle $\mathrm{F}$ makes a rapid deceleration to avoid a collision with the slower Vehicle L. The host vehicle's driver in this case has to decelerate to avoid a collision with Vehicle F.

The warning type support system sets off an auditory proximity warning when Vehicle $\mathrm{F}$ made a rapid deceleration.

The action type support system, on the other hand, applies an automatic panic brake when it detected a rapid deceleration of Vehicle F.

Each of 10 participants made a drive under Scenario 2 . They experienced firstly the baseline condition, and then two types of support functions. The order of presenting the warning type support condition and the action type support condition was counterbalanced.

Results. (a) Among 20 possibly hazardous occasions, seven collisions occurred under the baseline condition, two collisions under the warning type support, and no collision occurred under the action type support; see Table 3. McNemar test found significant difference only between the baseline condition and the action type support $(\mathrm{Z}=2.27, \mathrm{p}=$ 0.023 ).

(b) No significant difference was found on the subjective ratings of perceived efficacy in collision avoidance between the warning type and the action type support conditions; see Fig. 4.

(c) No significant difference was found, either, on the subjective ratings of system acceptance between the warning type and the action type; see Fig. 5.

(d) An ANOVA on the maximum brake pressure shows

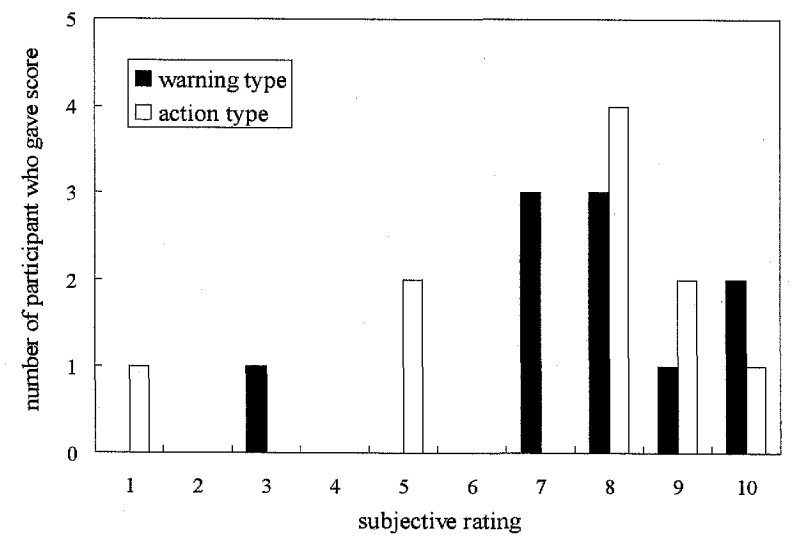

Fig. 5 Subjective ratings of acceptance of the support system under Scenario 2.

Table 4 Maximum brake pressure under Scenario 2.

\begin{tabular}{|c|c|c|c|c|c|}
\hline \multicolumn{6}{|c|}{ Maximum brake pressure $[\mathrm{Pa}]$} \\
\hline \multicolumn{2}{|c|}{$\begin{array}{l}\text { baseline } \\
\text { (no-aid) }\end{array}$} & \multicolumn{2}{|c|}{$\begin{array}{l}\text { warning } \\
\text { type }\end{array}$} & \multicolumn{2}{|c|}{$\begin{array}{c}\text { action } \\
\text { type }\end{array}$} \\
\hline M & $\mathrm{SD}$ & $\mathrm{M}$ & $\mathrm{SD}$ & M & SD \\
\hline 64.69 & 26.23 & 54.40 & 26.51 & 6.89 & 9.52 \\
\hline
\end{tabular}

a highly significant main effect of $\operatorname{LOA}(\mathrm{F}(2,16)=29.019$, $\mathrm{p}<0.00001$ ); see Table 4. No significant difference was found between the baseline and the warning type conditions. Tukey's HSD test revealed that the brake stroke was significantly smaller under the action type compared with any other condition $(\mathrm{p}<0.01)$.

\section{Discussions \& Conclusion}

The action type support system was not well accepted by the participants under Scenario 1, although it contributed appreciably to avoid collisions. The most prominent reason for it seemed to be conflict of intentions between the driver and the driver support system. When the "Lane Closed" indication was given, the driver usually decided to make a lane change. At that time, it was hard for the driver of the host vehicle to notice the approach of Vehicle $\mathrm{F}$ from behind, because Vehicle $\mathrm{F}$ was in the blind spot of the host vehicle. Changing a lane was thus a "natural and reasonable decision" for the host vehicle's driver. On the other hand, upon detecting the approach of Vehicle F, the driver support system determined that the host vehicle must stay in the cruising lane to avoid a possible collision against Vehicle F. That means that the driver and the driver support system formed different intentions, just because what the driver saw was quite different from what the support system saw. In the experiment reported in this paper, the driver support system cancelled out the driver's steering input without giving the driver any prior notice, which caused automation surprises. Most drivers did not understand what happened, when they saw their cars behaving "strangely." 
There would be two ways to resolve the automation surprise under Scenario 1. The first is to implement the envelope protection as a soft protection that makes the steering slightly heavier, instead of a hard protection that makes the steering extremely heavy or cancels out the driver's steering input completely. The envelope protection implemented in this paper was a hard protection.

The second is to set the LOA of envelope protection at 6.5 ; viz., the driver support system executes the action type support automatically, upon telling the driver what it is going to do. LOA 6.5 is more beneficial than any LOA at level 6 or lower, in avoiding time-delay before a safety critical protective action is executed, and is more promising than any LOA at level 7 or higher, in reducing automation surprises [26].

Participants appreciated and accepted the action type support under Scenario 2, in which they were not given the final authority over the automation. Moreover, result (d) under Scenario 2 shows that the driver could avoid a collision with less workload when the action type support was provided with, compared to the case when the driver support was of the warning type.

Combining the results under Scenario 1 with those under Scenario 2, it is argued that LOA must be situation and context adaptive [22]. In other words, in the case of an automobile, it is not sensible to assume that the driver must be maintained as the final authority at all times and on all occasion. Today's smart machine can sense, analyze situations, and decide what must be done. The results in this paper imply that such a smart machine may be given right to implement control actions so that it can help humans in a positive and proactive manner. Human-centered automation is an approach to realize a work environment in which humans and machines collaborate cooperatively, in which "humans" can vary considerably depending on the humanmachine system under consideration. It is needed to seek for a human-centered automation for automobile, instead of just importing a human-centered automation in aviation.

\section{Acknowledgments}

This study was conducted as part of the project, "Situation and Intention Recognition for Risk Finding and Avoidance" under the support of the Ministry of Education, Culture, Sports, Science and Technology, Government of Japan. The aim of the project is to develop a proactive safety technology that (i) detects possible transitions of the driver's psycholog$\mathrm{ical} / \mathrm{physiological}$ state into a risky condition that may lead to a possibly accident-prone driving condition, and (ii) provide the driver with appropriate and situation-adaptive countermeasure assistance and support.

\section{References}

[1] J.R. Treat, N.S. Tumbas, S.T. McDonald, D. Shinar, R.D. Hume, R.E. Mayer, R.L. Stansifer, and N.J. Castellan, "Tri-level study of the causes of traffic accidents: Final report," vol.1, Technical Report, Federal Highway Administration, US DOT, 1979.
[2] M. Green, What causes $90 \%$ of all automobile accidents? http://www.visualexpert.com/accidentcause.html

[3] E. Hollnagel and A. Bye, "Principles for modeling function allocation," Int. J. Human-Computer Studies, vol.52, pp.253-265, 2000.

[4] G.J. Witt, SAfety VEhicle(s) using adaptive interface technology (SAVE-IT) program, DTRS57-02-R-20003, 2004.

[5] M. Akamatsu, Y. Sakaguchi, and M. Okuwa, "Modeling of driving behavior when approaching an intersection based on measured behavioral data on an actual road," Proc. HFES 47th Annual Meeting, pp.1895-1899, 2003.

[6] F. Saad, "Studying behavioural adaptations to new driver support systems," Proc. Int'l Workshop on Modelling Driver Behaviour in Automotive Environments, pp.63-73, 2005.

[7] F. Tango and R. Montanari, "Modeling traffic and real situations," Proc. Int'1 Workshop on Modelling Driver Behaviour in Automotive Environments, pp.133-147, 2005.

[8] A. Amiditis, Z. Lentziou, A. Polychronopoulos, A. Bolovinou, and E. Bekiaris, "Real time traffic and environment monitoring for automotive applications," Proc. Int'l Workshop on Modelling Driver Behaviour in Automotive Environments, pp.125-131, 2005.

[9] M. Panou, E. Bekiaris, and V. Papakostopoulos, "Modeling driver behavior in EU and internationak projects," Proc. Int'l Workshop on Modelling Driver Behaviour in Automotive Environments, pp.5-21, 2005.

[10] T. Inagaki and M. Itoh, "Driver behavior monitoring: Part I. Application to adaptive automation implementation," Proc. DSC Asia/Pacific, CD-ROM 10 pages, 2006.

[11] M. Itoh, T. Akiyama, and T. Inagaki, "Driver behavior monitoring: Part II. Detection of driver's inattentiveness under distracting conditions," Proc. DSC Asia/Pacific, CD-ROM 10 pages, 2006.

[12] M. Itoh, H. Nagasaku, and T. Inagaki, "Analyses of driver's body movement for detection of hypovigilance due to non-driving cognitive tasks," Poc. IFAC CTS, CD-ROM 5 pages, 2006.

[13] D.D. Woods, "The effects of automation on human's role: Experience from non-aviation industries," in Flight Deck Automation: Promises and Realities, ed. S. Norman and H. Orlady, NASA CR10036, NASA-Ames Research Center, pp.61-85, 1989.

[14] C.E. Billings, Aviation automation - The search for a humancentered approach, LEA, 1997.

[15] T.B. Sheridan, Humans and Automation: System Design and Research Issues, Human Factors and Ergonomics Society \& Wiley, 2002.

[16] M.W. Scerbo, "Theoretical perspectives on adaptive automation," in Automation and Human Performance, ed. R. Parasuraman and M. Mouloua, pp.37-63, LEA, 1996.

[17] T. Inagaki, "Adaptive automation: Sharing and trading of control," in Handbook of Cognitive Task Design, ed. E. Hollnagel, pp.147-169, LEA, 2003.

[18] N.B. Sarter and D.D. Woods, "How in the world did we ever get into that mode? Mode error and awareness in supervisory control," Human Factors, vol.37, no.1, pp.5-19, 1995.

[19] R. Parasuraman and V. Riley, "Humans and automation: Use, misuse, disuse, abuse," Human Factors, vol.39, no.2, pp.230-253, 1997.

[20] N.B. Sarter, D.D. Woods, and C.E. Billings, "Automation surprises," in Handbook of Human Factors and Ergonomics, 2nd ed., ed. G. Salvendy, pp.1926-1943, Wiley, 1997.

[21] T. Inagaki and J. Stahre, "Human supervision and control in engineering and music: Similarities, dissimilarities, and their implications," Proc. IEEE, vol.92, no.4, pp.589-600, 2004.

[22] T. Inagaki, "Design of human-machine interactions in light of domain-dependence of human-centered automation," Cognition Technology \& Work, vol.8, no.3, pp.161-167, 2006.

[23] ICAO, Human factors training manual, Doc 9683-AN/950, 1998.

[24] T.B. Sheridan, Telerobotics, automation, and human supervisory control, MIT Press, 1992.

[25] T. Inagaki, N. Moray, and M. Itoh, "Trust self-confidence and authority in human-machine systems," Proc. IFAC Man-Machine Sys- 
tems, pp.431-436, 1998.

[26] T. Inagaki, H. Furukawa, and M. Itoh, "Human interaction with adaptive automation: Strategies for trading of control under possibility of over-trust and complacency," Proc. HCI- AugCog International, CD-ROM 10 pages, 2005.

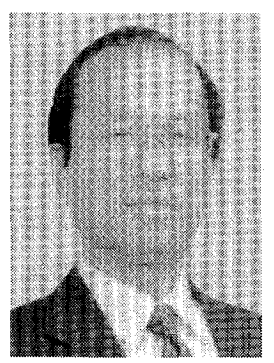

Toshiyuki Inagaki received B.S., M.S., and $\mathrm{Ph} . \mathrm{D}$. degrees in systems engineering from Kyoto University in 1974, 1976, and 1979, respectively. From 1979 to 1980 he was a research associate at the University of Houston. In 1980, he joined the University of Tsukuba, where he has been a professor since 1994. From 1990 to 1991 he was at the University of Kassel as a research fellow of the Alexander von Humboldt Foundation. His research interests include adaptive automation, human-computer interaction, trust in and distrust of automated systems, decision making under uncertainty with time stress. Dr. Inagaki is a senior member of IEEE, and a member of societies such as HFES, REAJ, SICE, ISCIE, JSAE, HIS. He served as chairs of the SICE SIG on Human-Machine Systems as well as the IEEE Reliability Society Japan Chapter. He received the best paper awards from the ISCIE in 1994, the HIS in 2001, and the best presentation award of JSAE in 2004.

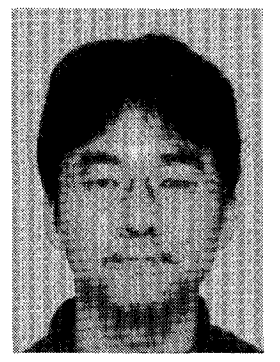

Makoto Itoh received B.S., M.S., and Ph.D. degrees from the University of Tsukuba in 1993, 1995 , and 1999, respectively. He was a research associate at the University of Tsukuba from 1996 to 1998, and at the University of ElectroCommunications from 1998 to 2002. He has been an assistant professor at the University of Tsukuba since 2002. His research interests include design and analysis of driver assist systems, trust and over-trust in automation, decision making under uncertainty. Dr. Itoh is a member of societies such as IEEE, HFES, SICE, HIS, JSAE, JSQC. He is chair of the SICE SIG on Human-Machine Systems, and served as secretary of the IEEE Reliability Society Japan Chapter. He received the best paper awards from HIS in 2001, SPEJ in 2002, academic encouraging award from HIS in 2002, Inoue Research Award for Young Scientists in 2000, and QC award from JUSE in 2002.

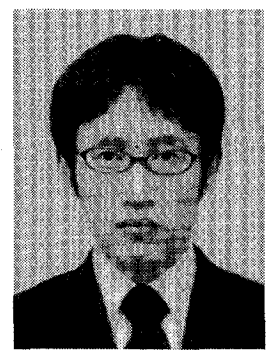

Yoshitomo Nagai received his B.S. degree from the University of Tsukuba in 2006, and is now working for his master's degree at the Graduate School of Systems and Information Engineering, University of Tsukuba. His research interests include design and implementation of advanced driver assist systems via an adaptive automation approach. Mr. Nagai is a member of JSAE. 